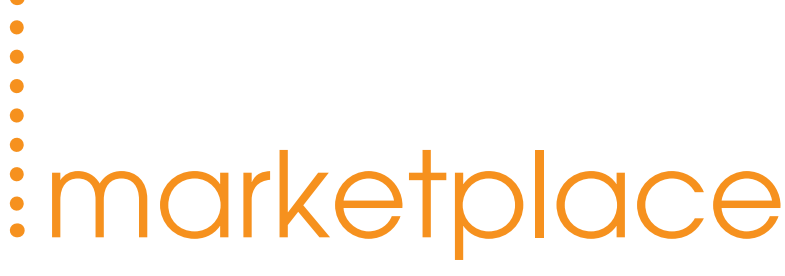

Marketplace is provided as a service to readers using text and images from the manufacturer, supplier or distributor and does not imply endorsement by Vital. Normal and prudent research should be exercised before purchase or use of any product mentioned.

\section{One stop dental shop}

Kent Express supply a wide selection of high quality oral health products, both from their own Kent Dental range as well as brand names from leading manufacturers, including Braun, Oral B, Colgate, Glaxo Smith Kline and TePe.

Kent Express is the 'one stop' dental supplier who can meet all of your practice requirements, making purchasing simpler and quicker. Orders can be placed online using Kent Connect for ease and speed, providing rapid access to the vast range of Kent Express products, news bulletins, special offers and promotions. For more information on Kent Express products call 01634878787.

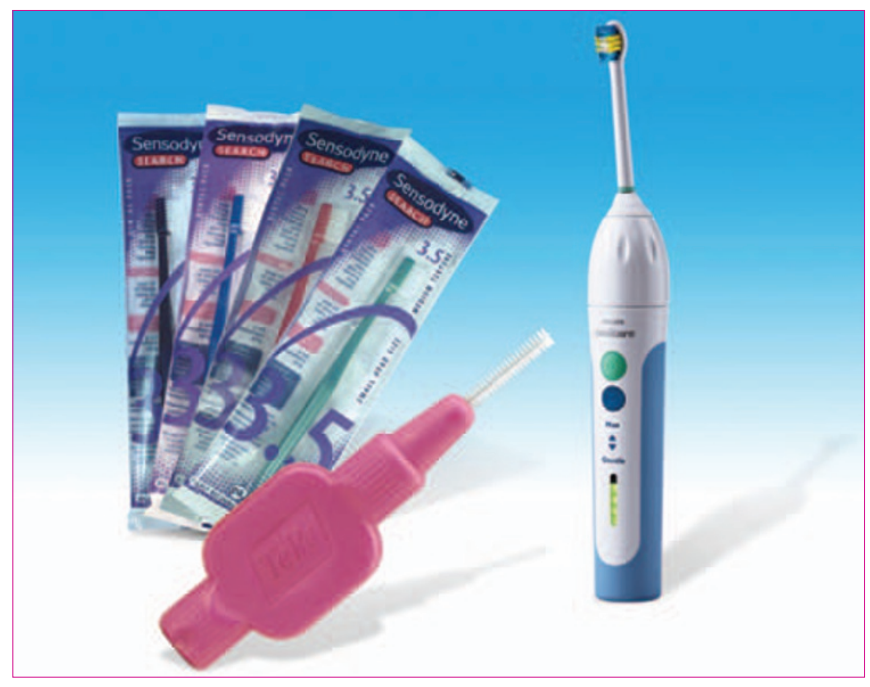

\section{Fluoride friendly floss}

Flix is a new disposable interdental stick that delivers fluoride when it comes into contact with saliva. Patented technology is used to attach a fine woven mesh of floss to each stick, which releases the fluoride.

With its tapered design and ergonomic handle, Flix is the ideal solution for cleaning interdentally, especially around orthodontic braces and implants. It is available in patient packs of 100 sticks and chairside packs of 500 (idea

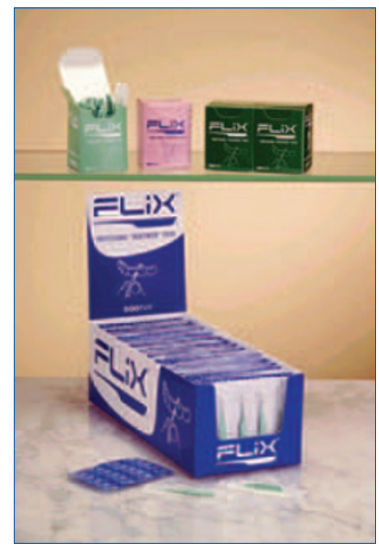
for patients to sample), and in 'Traditional', 'Mint Sensation' and 'Zero' (fluoride free) varieties. For further information or to order, please call Mirage Dental on 08451305440.

\section{Grit your sensitive teeth}

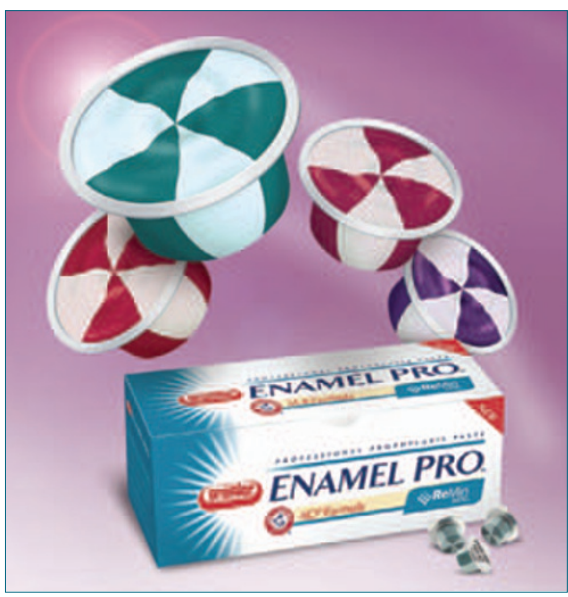

Myerson has announced the availability of their new ACP Prophy Paste, Enamel Pro from Premier. ACP (amorphous calcium phosphate) has been proven to stimulate the remineralisation of tooth enamel, helping to prevent tooth decay and reduce sensitivity. When applied to the tooth structure Enamel Pro releases ACP which remains in the tooth tissue even after rinsing. This ongoing remineralisation of the tooth reduces the incidence of caries. Despite containing the same percentage of fluoride (1.23\%) as conventional pastes, the ACP technology delivers $31 \%$ more fluoride than other prophy pastes, as the ACP increases the uptake of fluoride.

In addition to these benefits, Enamel Pro is designed to fill in surface crevices giving an intense polish and higher lustre for whiter, brighter teeth.

The Enamel Pro cups are competitively priced and supplied in four flavours: cinnamon, grape, strawberry and mint; and in fine, medium and coarse grits. The mint variety is also available in extra-coarse for heavier staining.

To order or for further information, contact Myerson Limited on 02089012828.

\section{Terrific trays}

Dentsply Ash Instruments manufacture Polytrays: high quality, bright and sturdy plastic single-use impression trays. Available in three colour-coded sizes for accurate fit, Ash Polytrays are compatible with all alginate and elastomeric impression materials and their adhesives.

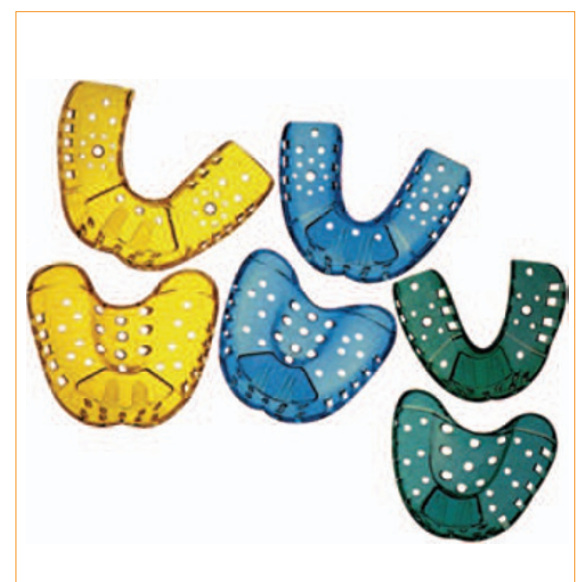

Polytrays are sup

plied in boxes of 25 of one type, or in an assorted pack containing four trays of each, in three sizes, upper and lower. Lower trays can be sectioned and used for small partials and the handle and nylon adapter are supplied. For more details, or to talk to your local Dentsply product specialist, please call 01932853422. 